\title{
Model to Predict Cause-Specific Mortality in Patients with Head and Neck Adenoid Cystic Carcinoma: A Competing Risk Analysis
}

\author{
Matin Imanguli, MD, DDS and Baran D. Sumer, MD \\ Department of Otolaryngology-Head and Neck Surgery, University of Texas Southwestern Medical Center, Dallas, TX
}

Adenoid cystic carcinoma is a relatively uncommon malignancy constituting approximately $1 \%$ of head and neck malignancies and approximately $10 \%$ of salivary gland tumors. ${ }^{1}$ It is the most common malignant tumor of the minor salivary glands and can occur throughout the head and neck, including in the major and minor salivary glands, nasal cavities and lacrimal glands. It is characterized by an indolent growth pattern with lymph node metastasis being uncommon, occurring only in approximately $10-15 \%$ of cases. Yet, despite this indolent biological behavior, it is classified as a high grade malignancy, primarily due to its propensity to recur locally despite clear surgical margins.

Surgery is the mainstay of treatment, with complete excision especially of early-stage tumors leading to an excellent prognosis. Schroeder et al. looking at various parotid gland malignancies in a retrospective trial, reported a 5 year disease free survival of $86 \%$. Patients with more advanced stage disease had a poorer prognosis. ${ }^{2}$ Risk factors for recurrence include bone invasion and positive margins as well as T3 or T4 disease. ${ }^{3}$ Distant metastasis, predominantly to the lungs, sometimes decades following excision of the primary, is very common and is often the cause of death. Early perineural invasion, one of the defining characteristics of this tumor, is thought to be responsible for spread outside grossly and microscopically visible margins.

Because of its low incidence, randomized prospective studies have been difficult to perform. Importantly, in contrast to most head and neck malignancies, short-term

(C) Society of Surgical Oncology 2017

First Received: 5 April 2017;

Published Online: 26 May 2017

B. D. Sumer, MD

e-mail: Baran.Sumer@UTSouthwestern.edu survival does not correlate with long-term survival as late recurrences are the rule rather than exception. While surgery for a resectable primary tumor, followed by radiation therapy is the current accepted standard of care, no prospective data exist to objectively assess the contribution of adjuvant radiotherapy to long-term survival. Data supporting the use of adjuvant radiation are limited to retrospective reviews showing improved local control. ${ }^{3-6}$ Even less evidence exists to support primary or adjuvant chemotherapy although the currently enrolling NRG 1008 trial, "A Randomized Phase II/III Study of Adjuvant Concurrent Radiation and Chemotherapy versus Radiation Alone in Resected High-Risk Malignant Salivary Gland Tumors" is designed to shed light on this question. ${ }^{7}$

In the absence of prospective data, studies of long-term survival have been limited to population-based database investigations, such as the one by Shen et al published in this issue of Annals of Surgical Oncology. The authors performed competing risks analysis and constructed a nomogram of disease specific survival of adenoid cystic carcinoma of the head and neck. Such analysis is particularly important given long term survival and late recurrences that increase the chances of death from unrelated causes.

Several previous studies examining survival of adenoid cystic carcinoma have been completed in both the US and internationally. A study by Ellington et al. similarly used a Surveillance, Epidemiology, and End Results (SEER) database but focused on an earlier time period. In that study, 5-, 10- and 15 year overall survival was 90.3, 79.9, and $69.2 \%$, respectively, with female sex, absence of distant disease, married status, oral primary and surgical treatment being associated with improved survival. ${ }^{8}$ A large European population study of 2611 patients similarly found female sex, younger age and oral cavity primary subsite to be predictive of improved survival, i.e. 78 and $65 \%$ at 5 and 10 years, respectively. ${ }^{9}$ A smaller 
Danish population study found 10 year overall and disease specific survival to be 58 and $75 \%$ respectively. ${ }^{10}$ As in previous studies, radiation therapy did not appear to improve overall survival, likely due to delayed development of distant disease. Finally, authors of an international study of 438 patients constructed nomograms for overall and cause specific survival. ${ }^{11}$ In this well-designed study using training and validation cohorts, female sex, younger age, earlier $\mathrm{T}$ stage, absence of distant disease and lymph node involvement, negative margins and oral cavity primaries were associated with improved survival.

The strengths of the study by Shen et al. stem from a large sample size and contemporary data that are likely to better reflect current trends in management. Focus on cause specific survival is important given the unique natural history of disease. Most of the variables included in the model have been previously identified as prognostically significant in other large population studies. In this paper, the authors create a competing risk model and nomogram to predict the probability of survival that is unique for adenoid cystic cancer. It is curious that radiation therapy was negatively associated with survival, however this could be because unresectable tumors and those with positive margins (information not available through the SEER database) were more likely to be irradiated. Longer follow-up and a validation cohort would help strengthen conclusions of the study as the median follow-up of 58 months is relatively short for this slow growing cancer. In addition since this is a SEER database trial histopathologic detail that may provide prognostic information is lacking. Finally, well designed, multicenter long-term prospective studies are needed to clarify the optimal approach in the management of adenoid cystic carcinoma.

\section{REFERENCES}

1. Coca-Pelaz A, Rodrigo JP, Bradley PJ et al. Adenoid cystic carcinoma of the head and neck-An update. Oral Oncol 2015; 51: 652-661.

2. Schroeder U, Groppe D, Mueller RP, Guntinas-Lichius O. Parotid cancer: Impact of changes from the 1997 to the 2002 American Joint Committee on Cancer classification on outcome prediction. Cancer 2008; 113: 758-764.

3. Roesink JM, Moerland MA, Hoekstra A et al. Scintigraphic assessment of early and late parotid gland function after radiotherapy for head-and-neck cancer: a prospective study of dosevolume response relationships. Int J Radiat Oncol Biol Phys 2004; 58: 1451-1460.

4. Garden AS, el-Naggar AK, Morrison WH et al. (1997) Postoperative radiotherapy for malignant tumors of the parotid gland. Int J Radiat Oncol Biol Phys 37: 79-85.

5. Armstrong JG, Harrison LB, Spiro RH et al. Observations on the natural history and treatment of recurrent major salivary gland cancer. J Surg Oncol 1990; 44: 138-141.

6. Harrison LB, Armstrong JG, Spiro RH et al. Postoperative radiation therapy for major salivary gland malignancies. J Surg Oncol 1990; 45: 52-55.

7. Dillon PM, Chakraborty S, Moskaluk CA et al. Adenoid cystic carcinoma: A review of recent advances, molecular targets, and clinical trials. Head Neck 2016; 38: 620-627.

8. Ellington CL, Goodman M, Kono SA et al. Adenoid cystic carcinoma of the head and neck: Incidence and survival trends based on 1973-2007 Surveillance, Epidemiology, and End Results data. Cancer 2012; 118: 4444-4451.

9. Ciccolallo L, Licitra L, Cantu G et al. Survival from salivary glands adenoid cystic carcinoma in European populations. Oral Oncol 2009; 45: 669-674.

10. Bjorndal K, Krogdahl A, Therkildsen $\mathrm{MH}$ et al. Salivary adenoid cystic carcinoma in Denmark 1990-2005: Outcome and independent prognostic factors including the benefit of radiotherapy. Results of the Danish Head and Neck Cancer Group (DAHANCA). Oral Oncol 2015; 51: 1138-1142.

11. Ganly I, Amit M, Kou L et al. Nomograms for predicting survival and recurrence in patients with adenoid cystic carcinoma. An international collaborative study. Eur J Cancer 2015; 51: 2768-2776. 